\title{
Phytoprotection
}

\section{Évaluation de l'activité antibactérienne contre Xanthomonas campestris pv. vitians et Pseudomonas cichorii de différents extraits végétaux à base d'espèces horticoles et d'essences forestières}

\section{Antibacterial activity against Xanthomonas campestris pv. vitians and Pseudomonas cichorii of different plant extracts prepared from horticultural and forest species}

\author{
Maxime Delisle-Houde, Vicky Toussaint et Russell J. Tweddell
}

Volume 99, numéro 1, 2019

Reçu 2019-02-25; accepté 2019-04-19

URI : https://id.erudit.org/iderudit/1062449ar

DOI : https://doi.org/10.7202/1062449ar

Aller au sommaire du numéro

Éditeur(s)

Société de protection des plantes du Québec (SPPQ)

ISSN

1710-1603 (numérique)

Découvrir la revue

Citer cet article

Delisle-Houde, M., Toussaint, V. \& Tweddell, R. J. (2019). Évaluation de l'activité antibactérienne contre Xanthomonas campestris pv. vitians et Pseudomonas cichorii de différents extraits végétaux à base d'espèces horticoles et d'essences forestières. Phytoprotection, 99(1), 21-26. https://doi.org/10.7202/1062449ar
Résumé de l'article

Différents extraits hydro-éthanoliques à base de résidus d'espèces horticoles et d'essences forestières ont été testés in vitro pour leur activité antibactérienne envers Xanthomonas campestris pv. vitians et Pseudomonas cichorii, respectivement responsables de la tache bactérienne et de la maladie des taches et des nervures noires de la laitue. Des 17 extraits d'espèces horticoles, 13 extraits affichent une concentration minimale létale (CML) $\geqslant 50 \mathrm{mg} \mathrm{ml}^{-1}$ envers $X$. campestris pv. vitians et $P$. cichorii. En contrepartie, trois des 20 extraits à base d'espèces forestières affichent une $\mathrm{CML} \geqslant 50 \mathrm{mg} \mathrm{ml}^{-1}$ envers les deux bactéries. De faibles CMLs $\left(\leqslant 6,25 \mathrm{mg} \mathrm{ml}^{-1}\right)$ envers les deux bactéries ont été obtenues avec l'extrait \#3 à base de fruits de canneberge et les extraits de fruits d'aulne rugueux, d'écorce de chêne rouge, d'écorce d'érable rouge et de rameaux de sapin baumier. Les résultats obtenus montrent que l'activité antibactérienne varie selon l'espèce et la structure utilisées dans la préparation de l'extrait; les extraits d'essences forestières présentant une plus forte activité antibactérienne. L'activité antibactérienne peut également varier selon le lot considéré d'un même extrait. Les extraits présentant une forte activité antibactérienne (CML $\leqslant 6,25 \mathrm{mg} \mathrm{ml}^{-1}$ ) seront testés au cours de travaux futurs afin d'évaluer leur efficacité à limiter le développement des populations de $X$. campestris pv. vitians et $P$. cichorii sur les plants de laitue et à réprimer le développement de la tache bactérienne et de la maladie des taches et des nervures noires.
Tous droits réservés @ La société de protection des plantes du Québec, 2019

Ce document est protégé par la loi sur le droit d'auteur. L'utilisation des services d'Érudit (y compris la reproduction) est assujettie à sa politique d'utilisation que vous pouvez consulter en ligne.

https://apropos.erudit.org/fr/usagers/politique-dutilisation/ 


\title{
Évaluation de l'activité antibactérienne contre Xanthomonas campestris pv. vitians et Pseudomonas cichorii de différents extraits végétaux à base d'espèces horticoles et d'essences forestières
}

\author{
Maxime Delisle-Houde ${ }^{1}$, Vicky Toussaint ${ }^{2}$ et Russell J. Tweddell ${ }^{1 \bowtie}$
}

Reçu 2019-02-25; accepté 2019-04-19

\section{PHYTOPROTECTION 99 : 21-26}

Différents extraits hydro-éthanoliques à base de résidus d'espèces horticoles et d'essences forestières ont été testés in vitro pour leur activité antibactérienne envers Xanthomonas campestris pv. vitians et Pseudomonas cichorii, respectivement responsables de la tache bactérienne et de la maladie des taches et des nervures noires de la laitue. Des 17 extraits d'espèces horticoles, 13 extraits affichent une concentration minimale létale $(\mathrm{CML}) \geq 50 \mathrm{mg} \mathrm{ml}^{-1}$ envers $X$. campestris pv. vitians et $P$. cichorii. En contrepartie, trois des 20 extraits à base d'espèces forestières affichent une $C M L \geq 50 \mathrm{mg} \mathrm{ml}^{-1}$ envers les deux bactéries. De faibles CMLs $\left(\leq 6,25 \mathrm{mg} \mathrm{ml}^{-1}\right)$ envers les deux bactéries ont été obtenues avec l'extrait \#3 à base de fruits de canneberge et les extraits de fruits d'aulne rugueux, d'écorce de chêne rouge, d'écorce d'érable rouge et de rameaux de sapin baumier. Les résultats obtenus montrent que l'activité antibactérienne varie selon l'espèce et la structure utilisées dans la préparation de l'extrait; les extraits d'essences forestières présentant une plus forte activité antibactérienne. L'activité antibactérienne peut également varier selon le lot considéré d'un même extrait. Les extraits présentant une forte activité antibactérienne $\left(\mathrm{CML} \leq 6,25 \mathrm{mg} \mathrm{ml}^{-1}\right)$ seront testés au cours de travaux futurs afin d'évaluer leur efficacité à limiter le développement des populations de $X$. campestris pv. vitians et $P$. cichorii sur les plants de laitue et à réprimer le développement de la tache bactérienne et de la maladie des taches et des nervures noires.

Mots-clés: activité antibactérienne, concentration minimale létale, extraits végétaux, Pseudomonas cichorii, Xanthomonas campestris pv. vitians.

[Antibacterial activity against Xanthomonas campestris pv. vitians and Pseudomonas cichorii of different plant extracts prepared from horticultural and forest species]

Different aqueous-ethanolic extracts prepared from wastes of horticultural and forest species were tested in vitro for their antibacterial activity against Xanthomonas campestris pv. vitians and Pseudomonas cichorii causing bacterial leaf spot and varnish spot of lettuce, respectively. Among the 17 horticultural extracts, 13 show minimum bactericidal concentrations (MBCs) $\geq 50 \mathrm{mg} \mathrm{ml}^{-1}$ against $X$. campestris pv. vitians and $P$. cichorii. On the other hand, three of the 20 forest extracts show MBCs $\geq 50 \mathrm{mg} \mathrm{ml}^{-1}$ against both bacteria. Low MBCs $\left(\leq 6.25 \mathrm{mg} \mathrm{ml}^{-1}\right)$ against both bacteria were obtained with extract \#3 from cranberry fruit, fruit extract from grey alder, bark extract from red oak, bark extract from red maple, and twig extract from balsam fir. Results obtained show that antibacterial activity varies according to species and structure used to prepare the extract; extracts from forest species exhibit stronger antibacterial activity. Antibacterial activity can also vary from batch to batch of a same extract. The extracts with strong antibacterial activity $\left(\mathrm{MBC} \leq 6.25 \mathrm{mg} \mathrm{m}^{-1}\right.$ ) will be further investigated for their efficacy to repress the development of $X$. campestris pv. vitians and $P$. cichorii populations on lettuce plants and to control bacterial leaf spot and varnish spot.

Keywords: antibacterial activity, minimum bactericidal concentration, plant extracts, Pseudomonas cichorii, Xanthomonas campestris pv. vitians.

1. Département de phytologie, Université Laval, Québec, QC, Canada G1V 0A6. Auteur de correspondance : russell.tweddell@fsaa.ulaval.ca.

2. Centre de recherche et de développement de Saint-Jean-sur-Richelieu, Agriculture et Agroalimentaire Canada, Saint-Jean-surRichelieu, QC, Canada J3B 3E6. 


\section{INTRODUCTION}

L'utilisation des pesticides conventionnels en agriculture est de plus en plus critiquée en raison de leurs nombreux impacts négatifs sur l'environnement (Guérin 2013; Hayes et al. 2006; Pimentel et al. 1992), la santé animale et la santé humaine (Alavanja et al. 2004; van der Werf 1996). Qui plus est, les pesticides disponibles sur le marché sont souvent inefficaces contre de nombreux agents phytopathogènes responsables de pertes économiques importantes, notamment contre plusieurs bactéries phytopathogènes. Il devient donc urgent de développer des produits efficaces de remplacement des pesticides conventionnels pour lutter contre les maladies affectant les plantes cultivées.

Parmi les différentes approches proposées pour réduire l'utilisation des pesticides conventionnels, l'exploitation à des fins phytosanitaires de résidus végétaux, souvent peu valorisés, soulève un intérêt grandissant auprès des scientifiques, des phytopathologistes et des différents intervenants du secteur agricole (Pino et al. 2013; Rguez et al. 2018). Les plantes représentent en effet une vaste source de molécules de différentes natures chimiques ayant des propriétés antimicrobiennes (Ncube et al. 2008), notamment des alcaloïdes, des composés phénoliques et des terpènes (Bennett et Wallsgrove 1994).

Dans cette étude, différents extraits à base de résidus d'espèces horticoles et d'essences forestières ont été testés in vitro afin d'estimer leur activité antibactérienne envers Xanthomonas campestris pv. vitians et Pseudomonas cichorii, bactéries phytopathogènes affectant la laitue (Lactuca sativa L.), utilisées comme bactéries modèles.

\section{MATÉRIEL ET MÉTHODES}

\section{$X$. campestris pv. vitians et $P$. cichorii}

Les souches virulentes B07-007 de $X$. campestris pv. vitians et B08-183 de P. cichorii de la collection de la Dre Vicky Toussaint (Agriculture et Agroalimentaire Canada, SaintJean-sur-Richelieu, QC, Canada) ont été utilisées. Les souches ont été conservées à $-85^{\circ} \mathrm{C}$ dans une solution de glycérol $15 \%$ (m/v) (VWR International, West Chester, PA, USA). Xanthomonas campestris pv. vitians a été cultivée à $28^{\circ} \mathrm{C}$ durant $48 \mathrm{~h}$ sur des géloses à base d'extrait de levures, de dextrose et de carbonate de calcium [YDC; $10 \mathrm{~g} \mathrm{~L}^{-1} \mathrm{~d}^{\prime}$ extrait de levures (Becton, Dickinson and Company, Sparks, MD, USA), $20 \mathrm{~g} \mathrm{~L}^{-1}$ de dextrose (Sigma-Aldrich Inc., St. Louis, MO, USA), $20 \mathrm{~g} \mathrm{~L}^{-1}$ de carbonate de calcium (Sigma-Aldrich Inc.) et $15 \mathrm{~g} \mathrm{~L}^{-1}$ d'agar-agar granulé (EM Science, Gibbstown, NJ, USA)]. Pseudomonas cichorii a été cultivée à $28^{\circ} \mathrm{C}$ durant $48 \mathrm{~h}$ sur des géloses Pseudomonas Agar F (PAF; Becton, Dickinson and Company) enrichies de glycérol $\left(10 \mathrm{~g} \mathrm{~L}^{-1}\right)$. Des suspensions bactériennes $\left[1 \times 10^{8}\right.$ unités formatrices de colonies (ufc) $\left.\mathrm{ml}^{-1}\right]$ ont été préparées dans une solution saline d'eau physiologique $(0,5 \% \mathrm{NaCl})$ selon la procédure décrite par Delisle-Houde et al. (2018) à partir des cultures sur géloses YDC (X. campestris pv. vitians) et PAF (P. cichorii).

\section{Extraits végétaux}

Différentes structures (aiguille, bulbe, écorce, feuille, fleuret, fruit, rameau et tige) d'espèces horticoles (Tableau 1) et d'essences forestières (Tableau 2) ont été broyées mécaniquement et macérées $(10-15 \%, \mathrm{~m} / \mathrm{v})$ sous agitation dans un mélange eau:éthanol (1:1, v:v) durant $2 \mathrm{~h}$ à température pièce.
Chacune des suspensions d'extraction a été évaporée à sec sous vacuum à une température $n^{\prime}$ excédant pas $60^{\circ} \mathrm{C}$ avec un évaporateur rotatif (Sigma-Aldrich Inc.). La poudre obtenue a été lyophilisée et conservée (obscurité) à température pièce dans des pots Mason.

\section{Concentrations minimales létales (CMLs) des extraits végétaux}

Les CMLs ont été déterminées sous conditions stériles à l'aide de microplaques de 96 puits (Sarstedt AG \& Co., Nümbrecht, Allemagne) selon le protocole décrit par Delisle-Houde et al. (2018). Les bactéries ( $X$. campestris pv. vitians ou $P$. cichorii) ont été mises en suspension $\left(5 \times 10^{5}\right.$ ufc) dans $100 \mu$ le bouillon nutritif de type King $B$ [ $10 \mathrm{~g} \mathrm{~L}^{-1}$ de Bacto ${ }^{\mathrm{TM}}$ Protéose Peptone No. 3 (Becton, Dickinson and Company), $0,75 \mathrm{~g} \mathrm{~L}^{-1}$ de phosphate de sodium dibasique (EM Science) et $5 \mathrm{~g} \mathrm{~L}^{-1}$ de glycérol] contenant différentes concentrations de chaque extrait végétal (0 $\mathrm{mg} \mathrm{ml}^{-1} ; 0,39 \mathrm{mg} \mathrm{ml}^{-1} ; 0,78 \mathrm{mg} \mathrm{ml}^{-1} ; 1,56 \mathrm{mg} \mathrm{ml}^{-1} ; 3,13 \mathrm{mg} \mathrm{ml}^{-1}$; $6,25 \mathrm{mg} \mathrm{ml}^{-1} ; 12,5 \mathrm{mg} \mathrm{ml}^{-1} ; 25 \mathrm{mg} \mathrm{ml}^{-1} ; 50 \mathrm{mg} \mathrm{ml}^{-1}$ ). Les microplaques ont été incubées à $28^{\circ} \mathrm{C}$ pour une période de $24 \mathrm{~h}$. Le contenu $(100 \mu \mathrm{l})$ de chacun des puits a ensuite été étalé sur gélose YDC ( $X$. campestris pv. vitians) ou PAF ( $P$. cichorii). Les géloses ont par la suite été incubées à $28^{\circ} \mathrm{C}$. Après $48 \mathrm{~h}$ d'incubation, elles ont été examinées pour la présence/absence de croissance bactérienne. Pour chaque extrait végétal, la concentration la plus faible à laquelle aucune croissance bactérienne n'a été observée correspondait à la CML. Chaque concentration a été testée en triplicat. Pour chacun des extraits, la CML a été déterminée à deux reprises (essais 1 et 2).

\section{RÉSULTATS}

Les CMLs des extraits à base d'espèces horticoles obtenues au cours de l'essai 1 sont présentées au Tableau 1. Les CMLs les plus faibles $\left(6,25 \mathrm{mg} \mathrm{ml}^{-1}\right)$ envers les deux bactéries à l'étude ont été obtenues avec l'extrait à base de fruits de canneberge (extrait \#3). L'extrait de bulbes d'oignon affiche une $\mathrm{CML}$ de $25 \mathrm{mg} \mathrm{ml}^{-1}$ pour $X$. campestris pv. vitians et de $12,5 \mathrm{mg} \mathrm{ml}^{-1}$ pour $P$. cichorii; tandis que l'extrait de fruits de houblon et l'extrait de fruits de bleuet (extrait \#1) présentent une $C M L$ de $25 \mathrm{mg} \mathrm{ml}^{-1}$ pour respectivement $X$. campestris pv. vitians et $P$. cichorii. Les autres extraits à base d'espèces horticoles accusent des CMLs $\geq 50 \mathrm{mg} \mathrm{ml}^{-1}$ pour les deux bactéries. Les résultats obtenus au cours de l'essai 2 sont en tous points identiques à ceux de l'essai 1.

Les CMLs des extraits à base d'espèces forestières déterminées au cours de l'essai 1 sont présentées au Tableau 2. Les CMLs les plus faibles envers $X$. campestris pv. vitians ont été obtenues avec les extraits à base de fruits d'aulne rugueux $\left(1,56 \mathrm{mg} \mathrm{ml}^{-1}\right)$, de rameaux de sapin baumier $\left(1,56 \mathrm{mg} \mathrm{ml}^{-1}\right)$, $\mathrm{d}^{\prime}$ aiguilles vertes $\left(1,56 \mathrm{mg} \mathrm{ml}^{-1}\right)$ et jaunes $\left(3,13 \mathrm{mg} \mathrm{ml}^{-1}\right)$ de mélèze laricin, d'écorce de bouleau blanc $\left(3,13 \mathrm{mg} \mathrm{ml}^{-1}\right)$, d'écorce de chêne blanc $\left(3,13 \mathrm{mg} \mathrm{ml}^{-1}\right)$, d'aiguilles $\left(6,25 \mathrm{mg} \mathrm{ml}^{-1}\right)$ et de rameaux $\left(6,25 \mathrm{mg} \mathrm{ml}^{-1}\right)$ d'épinette blanche, d'écorce de chêne rouge $\left(6,25 \mathrm{mg} \mathrm{m}^{-1}\right)$, d'écorce d'érable rouge $\left(6,25 \mathrm{mg} \mathrm{m}^{-1}\right)$ et d'écorce de pin gris $\left(6,25 \mathrm{mg} \mathrm{ml}^{-1}\right)$. Parmi ces extraits, les extraits à base de fruits d'aulne rugueux $\left(3,13 \mathrm{mg} \mathrm{ml}^{-1}\right)$, d'écorce de chêne rouge $\left(6,25 \mathrm{mg} \mathrm{m}^{-1}\right)$, d'écorce d'érable rouge $\left(6,25 \mathrm{mg} \mathrm{ml}^{-1}\right)$ et de rameaux de sapin baumier $\left(6,25 \mathrm{mg} \mathrm{ml}^{-1}\right)$ présentent également de faibles CMLs pour $P$. cichorii. Avec des CMLs $\geq 50 \mathrm{mg} \mathrm{mL}^{-1}$, les extraits d'écorce de pin blanc, d'écorce d'érable à sucre et d'écorce de sapin baumier affichent des CMLs parmi les plus élevées, et ce, pour les deux bactéries à l'étude. Les résultats obtenus au cours de l'essai 2 sont en tous points identiques à ceux de l'essai 1. 
Tableau 1. Concentration minimale létale (CML) pour Xanthomonas campestris pv. vitians et Pseudomonas cichorii de différents extraits à base d'espèces horticoles (essai 1)

\begin{tabular}{|c|c|c|c|}
\hline \multirow{2}{*}{ Espèce } & \multirow{2}{*}{ Structure utilisée ${ }^{a}$} & \multicolumn{2}{|c|}{$\mathrm{CML}\left(\mathrm{mg} \mathrm{ml} \mathrm{l}^{-1}\right)$} \\
\hline & & X. campestris pv. vitians & P. cichorii \\
\hline $\begin{array}{l}\text { Bleuet } \\
\text { Vaccinium myrtilloides Aiton }\end{array}$ & $\begin{array}{c}\text { Fruit extrait \#1 } \\
\text { Fruit extrait } \# 2 \\
\text { Fruit extrait \#3 } \\
\text { Feuille }\end{array}$ & $\begin{array}{c}>50^{\text {b }} ;>50^{c} ;>50^{d} \\
>50 ;>50 ;>50 \\
50 ; 50 ; 50 \\
50 ; 50 ; 50\end{array}$ & $\begin{array}{c}25 ; 25 ; 25 \\
>50 ;>50 ;>50 \\
50 ; 50 ; 50 \\
50 ; 50 ; 50\end{array}$ \\
\hline $\begin{array}{l}\text { Brocoli } \\
\text { Brassica oleracea L. }\end{array}$ & Fleuret & $50 ; 50 ; 50$ & $50 ; 50 ; 50$ \\
\hline $\begin{array}{l}\text { Canneberge } \\
\text { Vaccinium macrocarpon L. }\end{array}$ & $\begin{array}{l}\text { Fruit extrait \#1 } \\
\text { Fruit extrait \#2 } \\
\text { Fruit extrait \#3 }\end{array}$ & $\begin{array}{c}>50 ;>50 ;>50 \\
50 ; 50 ; 50 \\
6,25 ; 6,25 ; 6,25\end{array}$ & $\begin{array}{l}>50 ;>50 ;>50 \\
>50 ;>50 ;>50 \\
6,25 ; 6,25 ; 6,25\end{array}$ \\
\hline $\begin{array}{l}\text { Épinard } \\
\text { Spinacia oleracea L. }\end{array}$ & Feuille & $>50 ;>50 ;>50$ & $>50 ;>50 ;>50$ \\
\hline $\begin{array}{l}\text { Fraise } \\
\text { Fragaria xananassa Duchesne ex Rozier }\end{array}$ & $\begin{array}{c}\text { Feuille } \\
\text { Fruit extrait \#1 } \\
\text { Fruit extrait \#2 }\end{array}$ & $\begin{array}{l}>50 ;>50 ;>50 \\
>50 ;>50 ;>50 \\
>50 ;>50 ;>50\end{array}$ & $\begin{array}{l}>50 ;>50 ;>50 \\
>50 ;>50 ;>50 \\
>50 ;>50 ;>50\end{array}$ \\
\hline $\begin{array}{l}\text { Framboise } \\
\text { Rubus idaeus L. }\end{array}$ & Feuille & $>50 ;>50 ;>50$ & $>50 ;>50 ;>50$ \\
\hline $\begin{array}{l}\text { Houblon } \\
\text { Humulus lupulus L. }\end{array}$ & $\begin{array}{l}\text { Fruit } \\
\text { Feuille }\end{array}$ & $\begin{array}{l}25 ; 25 ; 25 \\
50 ; 50 ; 50\end{array}$ & $\begin{array}{l}50 ; 50 ; 50 \\
50 ; 50 ; 50\end{array}$ \\
\hline $\begin{array}{l}\text { Oignon } \\
\text { Allium cepa L. }\end{array}$ & Bulbe & $25 ; 25 ; 25$ & 12,$5 ; 12,5 ; 12,5$ \\
\hline $\begin{array}{l}\text { Poireau } \\
\text { Allium ampeloprasum L. }\end{array}$ & Tige et feuille & $50 ; 50 ; 50$ & $50 ; 50 ; 50$ \\
\hline
\end{tabular}

\footnotetext{
a Le matériel végétal a été macéré dans un mélange eau:éthanol (1:1, v:v).

b, c, d Réplicat 1, 2, 3.
}

\section{DISCUSSION}

L'exploitation des propriétés antimicrobiennes des molécules végétales pour lutter contre les bactéries pathogènes affectant I'homme ou les plantes cultivées soulève de plus en plus d'intérêt au sein de la communauté scientifique. Parmi les raisons expliquant cet intérêt grandissant figurent les effets indésirables de certains antibiotiques, agents de conservation ou autres composés antibactériens sur la santé humaine ou l'environnement, l'absence de composés efficaces contre plusieurs bactéries pathogènes et la perte d'efficacité (en raison de l'émergence de souches résistantes) de nombreux antibiotiques communément utilisés (Mostafa et al. 2018; Niamsa et Sittiwet 2009; O'Gara et al. 2000). La présente étude s'inscrit dans ce contexte. Elle avait pour objectif d'évaluer l'activité antibactérienne, via la détermination in vitro des CMLs, de différents extraits à base d'espèces horticoles ou forestières envers les bactéries phytopathogènes à Gram négatif $P$. cichorii et $X$. campestris pv. vitians.
Parmi les 37 extraits évalués dans cette étude, 17 étaient à base d'espèces horticoles alors que 20 étaient à base d'essences forestières. Des 17 extraits d'espèces horticoles, 13 extraits affichent une $C M L \geq 50 \mathrm{mg} \mathrm{ml}^{-1}$ envers $X$. campestris pv. vitians et $P$. cichorii (Tableau 1). En contrepartie, trois des 20 extraits à base d'espèces forestières affichent une $C M L \geq 50 \mathrm{mg} \mathrm{ml}^{-1}$ envers les deux bactéries (Tableau 2). Sur la base des CMLs et des espèces sélectionnées dans la présente étude, les extraits à base d'essences forestières présentent une plus forte activité bactéricide. Cette dernière pourrait être en partie expliquée par les fortes teneurs en tannins retrouvées généralement chez les espèces forestières (Kraus et al. 2003) puisque ces composés ont un fort pouvoir antimicrobien (Scalbert 1991).

L'activité antibactérienne varie non seulement selon l'espèce horticole ou forestière, mais également selon la structure considérée, et ce, pour l'une ou l'autre des bactéries. Les extraits à base de fruits et d'écorce d'aulne rugueux présentent des CMLs très différentes envers les deux bactéries (Tableau 2). De même, l'activité antibactérienne peut pour 
Tableau 2. Concentration minimale létale (CML) pour Xanthomonas campestris pv. vitians et Pseudomonas cichorii de différents extraits à base d'espèces forestières (essai 1)

\begin{tabular}{|c|c|c|c|}
\hline \multirow{2}{*}{ Espèce } & \multirow{2}{*}{ Structure utilisé $e^{a}$} & \multicolumn{2}{|c|}{$\mathrm{CML}\left(\mathrm{mg} \mathrm{m}^{-1}\right)$} \\
\hline & & X. campestris pv. vitians & P. cichorii \\
\hline $\begin{array}{l}\text { Aulne rugueux } \\
\text { Alnus incana subsp. rugosa (Du Roi) R.T. Clausen }\end{array}$ & $\begin{array}{l}\text { Écorce } \\
\text { Fruit }\end{array}$ & $\begin{array}{c}50^{\text {b }} ; 50^{c} ; 50^{d} \\
1,56 ; 1,56 ; 1,56\end{array}$ & $\begin{array}{c}25 ; 25 ; 25 \\
3,13 ; 3,13 ; 3,13\end{array}$ \\
\hline $\begin{array}{l}\text { Bouleau blanc } \\
\text { Betula papyrifera Marsh. }\end{array}$ & Écorce & 3,$13 ; 3,13 ; 3,13$ & $25 ; 25 ; 25$ \\
\hline $\begin{array}{l}\text { Chêne blanc } \\
\text { Quercus alba L. }\end{array}$ & Écorce & 3,$13 ; 3,13 ; 3,13$ & 12,$5 ; 12,5 ; 12,5$ \\
\hline $\begin{array}{l}\text { Chêne rouge } \\
\text { Quercus rubra } \mathrm{L} \text {. }\end{array}$ & Écorce & 6,$25 ; 6,25 ; 6,25$ & 6,$25 ; 6,25 ; 6,25$ \\
\hline $\begin{array}{l}\text { Épinette blanche } \\
\text { Picea glauca (Moench) Voss }\end{array}$ & $\begin{array}{l}\text { Aiguille } \\
\text { Rameau }\end{array}$ & $\begin{array}{l}6,25 ; 6,25 ; 6,25 \\
6,25 ; 6,25 ; 6,25\end{array}$ & $\begin{array}{l}25 ; 25 ; 25 \\
50 ; 50 ; 50\end{array}$ \\
\hline $\begin{array}{l}\text { Érable à sucre } \\
\text { Acer saccharum Marsh. }\end{array}$ & Écorce & $50 ; 50 ; 50$ & $50 ; 50 ; 50$ \\
\hline $\begin{array}{l}\text { Érable rouge } \\
\text { Acer rubrum L. }\end{array}$ & $\begin{array}{l}\text { Écorce } \\
\text { Feuille }\end{array}$ & $\begin{array}{l}6,25 ; 6,25 ; 6,25 \\
>50 ;>50 ;>50\end{array}$ & $\begin{array}{l}6,25 ; 6,25 ; 6,25 \\
12,5 ; 12,5 ; 12,5\end{array}$ \\
\hline $\begin{array}{l}\text { Sapin baumier } \\
\text { Abies balsamea (L.) Mill. }\end{array}$ & $\begin{array}{l}\text { Aiguille } \\
\text { Rameau } \\
\text { Écorce }\end{array}$ & $\begin{array}{l}12,5 ; 12,5 ; 12,5 \\
1,56 ; 1,56 ; 1,56 \\
>50 ;>50 ;>50\end{array}$ & $\begin{array}{c}25 ; 25 ; 25 \\
6,25 ; 6,25 ; 6,25 \\
50 ; 50 ; 50\end{array}$ \\
\hline $\begin{array}{l}\text { Mélèze laricin } \\
\text { Larix laricina (Du Roi) K. Koch }\end{array}$ & $\begin{array}{l}\text { Aiguille jaune } \\
\text { Aiguille verte } \\
\text { Écorce }\end{array}$ & $\begin{array}{l}3,13 ; 3,13 ; 3,13 \\
1,56 ; 1,56 ; 1,56 \\
12,5 ; 12,5 ; 12,5\end{array}$ & $\begin{array}{c}25 ; 25 ; 25 \\
12,5 ; 12,5 ; 12,5 \\
50 ; 50 ; 50\end{array}$ \\
\hline $\begin{array}{l}\text { Merisier } \\
\text { Prunus avium (L.) L. }\end{array}$ & Écorce & 12,$5 ; 12,5 ; 12,5$ & $25 ; 25 ; 25$ \\
\hline $\begin{array}{l}\text { Peuplier faux-tremble } \\
\text { Populus tremuloides Michx. }\end{array}$ & Écorce & $25 ; 25 ; 25$ & $50 ; 50 ; 50$ \\
\hline $\begin{array}{l}\text { Pin blanc } \\
\text { Pinus strobus L. }\end{array}$ & Écorce & $50 ; 50 ; 50$ & $50 ; 50 ; 50$ \\
\hline $\begin{array}{l}\text { Pin gris } \\
\text { Pinus banksiana Lamb. }\end{array}$ & Écorce & 6,$25 ; 6,25 ; 6,25$ & 12,$5 ; 12,5 ; 12,5$ \\
\hline
\end{tabular}

${ }^{a}$ Le matériel végétal a été macéré dans un mélange eau:éthanol (1:1, v:v).

b, c, d Réplicat 1, 2, 3.

un même extrait varier d'un lot à l'autre, et ce, bien que le procédé de préparation des extraits ait été le même. Par exemple, l'extrait \#2 et l'extrait \#3 de fruits de canneberge présentent respectivement une $C M L \geq 50 \mathrm{mg} \mathrm{ml}^{-1}$ et $6,25 \mathrm{mg} \mathrm{ml}^{-1}$ (Tableau 1). Ceci suggère que les composés à l'origine de l'activité antibactérienne ou leurs concentrations peuvent varier non seulement selon l'espèce et la structure considérées, mais également selon le lot considéré d'un extrait. Considérant que les propriétés antibactériennes de la canneberge sont en grande partie attribuables à leur teneur élevée en composés phénoliques et en anthocyanes (Côté et al. 2011; Nohynek et al. 2006), on peut supposer que l'extrait \#2 de fruits de canneberge présentait une teneur inférieure en composés phénoliques et en anthocyanes.

$L^{\prime}$ extrait \#3 à base de fruits de canneberge avec une CML de $6,25 \mathrm{mg} \mathrm{ml}^{-1}$ envers les deux bactéries testées et l'extrait à base de bulbes d'oignon avec une CML de $25 \mathrm{mg} \mathrm{ml}^{-1}$ envers $X$. campestris pv. vitians et de $12,5 \mathrm{mg} \mathrm{ml}^{-1}$ envers $P$. cichorii sont les deux extraits d'espèces horticoles montrant la plus 
forte activité antibactérienne. Plusieurs études rapportent l'activité antibactérienne des extraits de canneberge et d'oignon. À titre d'exemples, la canneberge est rapportée pour son activité antibactérienne envers Listeria monocytogenes, Salmonella Typhimurium (Wu et al. 2008), Streptococcus mutans (Yoo et al. 2011), Pseudomonas aeruginosa (Côté et al. 2011), Escherichia coli (Pérez-López et al. 2009) et Staphylococcus aureus (Su et al. 2012) et l'oignon envers Bacillus subtilis (Ye etal. 2013), E. coli, Streptococcus pneumoniae (Benmeddour et al. 2015), Helicobacter pylori (Ramos et al. 2006), Bacillus cereus, Micrococcus luteus, L. monocytogenes (Santas et al. 2010), Salmonella Enteritidis (Benkeblia 2004) et S. aureus (Benkeblia 2004; Benmeddour et al. 2015; Santas et al. 2010; Ye et al. 2013).

En ce qui a trait aux extraits d'essences forestières, quatre extraits (extraits de fruits d'aulne rugueux, d'écorce de chêne rouge, d'écorce d'érable rouge et de rameaux de sapin baumier) présentent une forte $\left(C M L \leq 6,25 \mathrm{mg} \mathrm{ml}^{-1}\right)$ activité antibactérienne envers les deux bactéries. Sept extraits (extraits d'écorce de bouleau blanc, d'écorce de chêne blanc, d'écorce de pin gris, d'aiguilles jaunes et vertes de mélèze laricin, d'aiguilles et de rameaux d'épinette blanche) ont montré une forte activité antibactérienne envers X. campestris pv. vitians. Les travaux de Cellini et al. (1996), réalisés avec 19 souches de la bactérie $H$. pylori, ont montré que les CMLs envers $H$. pylori d'extraits aqueux de bulbes d'ail sont de l'ordre de 2,5 à $5 \mathrm{mg} \mathrm{ml}^{-1}$. Considérant d'une part, que l'ail est reconnu pour ses propriétés antimicrobiennes (Bakri et Douglas 2005; O'Gara et al. 2000) et d'autre part, qu'il constitue l'ingrédient actif de plusieurs biopesticides homologués au Canada (Santé Canada 2019), il est intéressant de constater que plusieurs extraits forestiers montrent une activité bactéricide comparable à celles obtenues par Cellini et al. (1996) pour des extraits d'ail.

Les extraits d'essences forestières se sont en général montrés plus toxiques envers la bactérie $X$. campestris pv. vitians. Plusieurs des plantes ligneuses susmentionnées sont rapportées pour avoir manifesté une activité antibactérienne. Omar et al. (2000) ont, au moyen d'un test de diffusion sur agar, observé l'activité antibactérienne d'extraits à base d'écorce d'érable rouge, de bouleau blanc et de chêne rouge envers B. subtilis, Mycobacterium phlei et $S$. aureus. Au moyen de ce même test, Lindberg et al. (2004) ont observé la toxicité d'un extrait de pin gris envers Bacillus coagulans, Burkholderia multivorans et Alcaligenes xylosoxidans. Des extraits de sapin baumier et de pin gris ont montré une activité antibactérienne (faible concentration minimale inhibitrice) envers $S$. aureus (Vandal et al. 2015). Les structures utilisées (aiguilles) par Vandal et al. (2015) pour préparer les extraits étaient toutefois différentes de celles utilisées dans la présente étude. Différents extraits d'aulne glutineux (Alnus glutinosa (L.) Gaertn.), une espèce très proche de l'aulne rugueux, sont rapportés dans la littérature pour leur activité antibactérienne (Abedini et al. 2016; Middleton et al. 2005). Les extraits à base de semences ont inhibé plusieurs bactéries dont Citrobacter freundii, E. coli, P. aeruginosa et S. aureus (Middleton et al. 2005) et ceux à base d'écorce une multitude d'espèces à Gram positif et à Gram négatif (Abedini et al. 2016).

Plusieurs extraits d'espèces horticoles ou forestières testés dans le cadre de cette étude montrent une forte activité antibactérienne envers $X$. campestris pv. vitians et $P$. cichorii, bactéries respectivement responsables de la tache bactérienne et de la maladie des taches et des nervures noires de la laitue (Toussaint 2008). Toutefois, pour être exploités à des fins phytosanitaires, les extraits végétaux doivent exercer une activité antibactérienne in planta. Les extraits présentant une forte activité antibactérienne seront donc testés au cours de travaux futurs afin d'évaluer leur efficacité à limiter le développement des populations de $X$. campestris pv. vitians et $P$. cichorii sur les plants de laitue et à réprimer le développement de la tache bactérienne et de la maladie des taches et des nervures noires. Des travaux additionnels seront également réalisés avec une large gamme de bactéries afin d'estimer l'étendue du spectre antibactérien des extraits ayant montré une forte toxicité envers $X$. campestris pv. vitians et $P$. cichorii.

\section{REMERCIEMENTS}

Les auteurs remercient le ministère de l'Agriculture, des Pêcheries et de l'Alimentation du Québec pour le support financier. Ils remercient également le Centre de recherche industrielle du Québec (CRIQ) pour avoir offert les nombreux extraits à base d'essences forestières, produits dans le cadre d'un projet du CRIQ financé par le ministère des Forêts, de la Faune et des Parcs du Québec.

\section{RÉFÉRENCES}

Abedini, A., S. Chollet, A. Angelis, N. Borie, J.-M. Nuzillard, A.-L. Skaltsounis, R. Reynaud, S.C. Gangloff, J.-H. Renault et J. Hubert. 2016. Bioactivity-guided identification of antimicrobial metabolites in Alnus glutinosa bark and optimization of oregonin purification by Centrifugal Partition Chromatography. J. Chromatogr. B 1029-1030 : 121-127.

Alavanja, M.C.R., J.A. Hoppin et F. Kamel. 2004. Health effects of chronic pesticide exposure: cancer and neurotoxicity. Annu. Rev. Public Health 25 : 155-197.

Bakri, I.M. et C.W.I. Douglas. 2005. Inhibitory effect of garlic extract on oral bacteria. Arch. Oral Biol. 50 : 645-651.

Benkeblia, N. 2004. Antimicrobial activity of essential oil extracts of various onions (Allium cepa) and garlic (Allium sativum). Lebensm.-Wiss. Technol. 37 : 263-268.

Benmeddour, T., H. Laouer, A.A. Benabdi et S. Brahimi. 2015. Évaluation de l'activité antibactérienne et antifongique des extraits de trois espèces du genre Allium : A. cepa, fistulosum et sativum cultivées dans le périmètre agricole de Doussen (Wilaya de Biskra). Courrier du savoir 19:9-14.

Bennett, R.N. et R.M. Wallsgrove. 1994. Secondary metabolites in plant defense mechanisms. New Phytol. 127 : 617-633.

Cellini, L., E. Di Campli, M. Masulli, S. Di Bartolomeo et N. Allocati. 1996. Inhibition of Helicobacter pylori by garlic extract (Allium sativum). FEMS Immunol. Med. Mic. 13 : 273-277.

Côté, J., S. Caillet, G. Doyon, D. Dussault, J.-F. Sylvain et M. Lacroix. 2011. Antimicrobial effect of cranberry juice and extracts. Food Control 22 : 1413-1418.

Delisle-Houde, M., V. Toussaint, H. Affia et R.J. Tweddell. 2018. Evaluation of different salts for the control of lettuce varnish spot: when phytotoxicity rules. Can. J. Plant Sci. $98: 753-761$.

Guérin, M. 2013. Introduction - La réduction des pesticides agricoles. Enjeux, modalités et conséquences. Économie Rurale 333 : 7-9.

Hayes, T.B., P. Case, S. Chui, D. Chung, C. Haeffele, K. Haston, M. Lee, V.P. Mai, Y. Marjuoa, J. Parker et M. Tsui. 2006. Pesticide mixtures, endocrine disruption, and amphibian declines: are we underestimating the impact? Environ. Health Persp. 114 : 40-50.

Kraus, T.E.C., R.A. Dahlgren et R.J. Zasoski. 2003. Tannins in nutrient dynamics of forest ecosystems - a review. Plant and Soil $256: 41-66$. 
Lindberg, L.E., S.M. Willför et B.R. Holmbom. 2004. Antibacterial effects of knotwood extractives on paper mill bacteria. J. Ind. Microbiol. Biot. 31 : 137-147.

Middleton, P., F. Stewart, S. Al-Qahtani, P. Egan, C. O'Rourke, A. Abdulrahman, M. Byres, M. Middleton, Y. Kumarasamy, M. Shoeb, L. Nahar, A. Delazar et S.D. Sarker. 2005. Antioxidant, antibacterial activities and general toxicity of Alnus glutinosa, Fraxinus excelsior and Papaver rhoeas. Iran. J. Pharm. Res. 4 : 101-103.

Mostafa, A.A., A.A. Al-Askar, K.S. Almaary, T.M. Dawoud, E.N. Sholkamy et M.M. Bakri. 2018. Antimicrobial activity of some plant extracts against bacterial strains causing food poisoning diseases. Saudi J. Biol. Sci. 25 : 361-366.

Ncube, N.S., A.J. Afolayan et A.I. Okoh. 2008. Assessment techniques of antimicrobial properties of natural compounds of plant origin: current methods and future trends. Afr. J. Biotechnol. 7 : 1797-1806.

Niamsa, N. et C. Sittiwet. 2009. Antimicrobial activity of Curcuma longa aqueous extract. J. Pharmacol. Toxicol. $4: 173-177$.

Nohynek, L.J., H.-L. Alakomi, M.P. Kähkönen, M. Heinonen, I.M. Helander, K.-M. Oksman-Caldentey et R.H. PuupponenPimiä. 2006. Berry phenolics: antimicrobial properties and mechanisms of action against severe human pathogens. Nutrition and Cancer 54 : 18-32.

O'Gara, E.A., D.J. Hill et D.J. Maslin. 2000. Activities of garlic oil, garlic powder, and their diallyl constituents against Helicobacter pylori. Appl. Environ. Microb. 66 : 2269-2273.

Omar, S., B. Lemonnier, N. Jones, C. Ficker, M.L. Smith, C. Neema, G.H. Towers, K. Goel et J.T. Arnason. 2000. Antimicrobial activity of extracts of eastern North American hardwood trees and relation to traditional medicine. J. Ethnopharmacol. $73: 161-170$.

Pérez-López, F.R., J. Haya et P. Chedraui. 2009. Vaccinium macrocarpon: An interesting option for women with recurrent urinary tract infections and other health benefits. J. Obstet. Gynaecol. Res. 35 : 630-639.

Pimentel, D., H. Acquay, M. Biltonen, P. Rice, M. Silva, J. Nelson, V. Lipner, S. Giordano, A. Horowitz et M. D’Amore. 1992. Environmental and economic costs of pesticide use. BioScience 42 : 750-760.

Pino, O., Y. Sánchez et M.M. Rojas. 2013. Plant secondary metabolites as an alternative in pest management. I: Background, research approaches and trends. Rev. Protección Veg. 28 : 81-94.
Ramos, F.A., Y. Takaishi, M. Shirotori, Y. Kawaguchi, K. Tsuchiya, H. Shibata, T. Higuti, T. Tadokoro et M. Takeuchi. 2006. Antibacterial and antioxidant activities of quercetin oxidation products from yellow onion (Allium cepa) skin. J. Agric. Food Chem. 54 : 3551-3557.

Rguez, S., N. Djébali, I.B. Slimene, G. Abid, M. Hammemi, S. Chenenaoui, S. Bachkouel, M. Daami-Remadi, R. Ksouri et I. Hamrouni-Sellami. 2018. Cupressus sempervirens essential oils and their major compounds successfully control postharvest grey mould disease of tomato. Ind. Crop Prod. 123 : 135-141.

Santas, J., M.P. Almajano et R. Carbó. 2010. Antimicrobial and antioxidant activity of crude onion (Allium cepa, L.) extracts. Int. J. Food Sci. Tech. 45 : 403-409.

Santé Canada. 2019. Recherche dans les étiquettes de pesticides. Agence de réglementation de la lutte antiparasitaire, Ottawa, ON. [En ligne] http://pr-rp.hcsc.gc.ca/ls-re/index-fra.php (consulté le 1 avril 2019).

Scalbert, A. 1991. Antimicrobial properties of tannins. Phytochemistry 30 : 3875-3883.

Su, X., A.B. Howell et D.H. D'Souza. 2012. Antibacterial effects of plant-derived extracts on methicillin-resistant Staphylococcus aureus. Foodborne Pathog. Dis. 9: 573-578.

Toussaint, V. 2008. Ma salade est malade, à qui la faute ? Le point sur les maladies bactériennes dans la laitue. Journées Horticoles de Saint-Rémi : petits fruits. Centre communautaire de Saint-Rémi, Saint-Rémi, QC. [En ligne] https://www.agrireseau.net/legumeschamp/document s/Toussaint\%20JH2008.pdf (consulté le 10 février 2019).

van der Werf, H.M.G. 1996. Assessing the impact of pesticides on the environment. Agric. Ecosyst. Environ. 60 : 81-96.

Vandal, J., M.M. Abou-Zaid, G. Ferroni et L.G. Leduc. 2015. Antimicrobial activity of natural products from the flora of Northern Ontario, Canada. Pharm. Biol. $53: 800-806$.

Wu, V.C.-H., X. Qiu, A. Bushway et L. Harper. 2008. Antibacterial effects of American cranberry (Vaccinium macrocarpon) concentrate on foodborne pathogens. Lebensm.-Wiss. Technol. 41 : 1834-1841.

Ye, C.-L., D.-H. Dai et W.-L. Hu. 2013. Antimicrobial and antioxidant activities of the essential oil from onion (Allium cepa L.). Food Control 30 : 48-53.

Yoo, S., R.M. Murata et S. Duarte. 2011. Antimicrobial traits of tea- and cranberry-derived polyphenols against Streptococcus mutans. Caries Res. 45 : 327-335. 